\title{
ELECCIONES AL PARLAMENTO EUROPEO DE 12 DE JUNIO DE 1994
}

\author{
JUAN MANUEL GOIG MARTÍNEZ \\ Profesor Asociado del Departamento de \\ Derecho Constitucional de la UNED
}




\section{SUMARIO}

I. Introducción.-II. La campaña electoral.-1. La Campaña en los Estados miembros.-2. La Campaña Electoral en España.-a) Los partidos y coaliciones en campaña.-b) Sondeos electorales.-III. Resultados electorales. - 1. Datos generales de participación.-2. Resultados por estados. - 3. Resultados en España.-a) Resultados generales.-b) Resultados por partidos y coaliciones.-IV. CONCLUSIONES. 
Revista de Derecho Político, núm. 41, 1996, pp. 251-268

\title{
ELECCIONES AL PARLAMENTO EUROPEO DE 12 DE JUNIO DE 1994
}

\author{
POR \\ JUAN MANUEL GOIG MARTINEZ \\ Profesor Asociado del Departamento de \\ Derecho Constitucional de la UNED
}

\section{INTRODUCCIÓN}

El día 12 de junio de 1994 se celebrarán, en la mayoria de los Estados miembros de la Unión Europea (en Irlanda, Holanda y Dinamarca la cita con las urnas es el 10 de junio) elecciones para la renovación total del Parlamento Europeo.

A pesar de que los problemas nacionales preocupan a los ciudadanos mucho más que los que se deciden en las Instituciones europeas, a partir de ahora las decisiones tomadas por el Parlamento Europeo nos van a afectar en mayor medida. Aunque seguirá sin poder aprobar leyes, podrá rechazar el presupuesto, en bloque, de la Unión, las partidas que no sean obligatorias (fondos de cohesión y financiación de la política agraria) e incluso el nombramiento del Presidente de la Comisión y su equipo de Comisarios.

Al igual que en los Parlamentos nacionales, los diputados se agrupan en fracciones según su ideología, pero para estas elecciones el número de escaños a cubrir aumenta de 518 a 567 . Sólo 3 Estados mantienen el número de eurodiputados: Irlanda (15), Dinamarca (16) y Luxemburgo (6). El resto aumenta sus representantes en la Cámara europea.

El 1 de enero de 1995, con la posible incorporación de Austria, Finlandia, Suecia y Noruega, el número de Parlamentarios puede ascender a 639 . 


\section{LA CAMPAÑA ELECTORAL}

\section{La Campaña en los Estados miembros}

Si estas elecciones al Parlamento Europeo constituyen un importante test en España, en relación a las elecciones Generales de 1995, otro tanto puede predicarse respecto a Dinamarca, donde los resultados electorales pueden ser considerados un ensayo general de las que se celebrarán en Diciembre.

Los resultados electorales pueden acabar en Gran Bretaña con el prestigio de John Major y provocar su sustitución al frente del Partido Conservador. Algo parecido a lo que puede suceder en Bélgica, donde la frágil coalición presidida por el democristiano Jean-Luc Dehaene podría resquebrajarse si no consigue más del 50 por 100 de los votos.

En el resto de Europa, la situación no alcanza altas cuotas de preocupación. En Holanda se celebran elecciones en el mes de abril, de forma que las europeas se celebran a escaso tiempo de diferencia, lo que supone que no haya grandes sorpresas. La coalición de democristianos y liberales que preside Helmut Kohl en alemania es la gran favorita, de forma que las elecciones europeas no son unas primarias para las generales que se celebrarán el día 16 de octubre. Silvio Berlusconi espera, en Italia, consolidarse como el político de mayor apoyo popular, y, en Grecia, el Primer Ministro Papandreu no corre peligro de un resultado adverso. Esta situación es parecida a la irlandesa, donde los comicios no se plantean como un barómetro de la política interna, y la coalición de nacionalistas, conservadores y laboristas, presumiblemente no se resentirá.

En Portugal, los posibles resultados adversos no pondrán en peligro al Gobierno centrista de Cavaco Silva, que goza de la mayoría absoluta, y en Francia, el centro-derecha presenta lista única, con lo que, seguramente, obtendrá unos buenos resultados que pueden mejorar las posibilidades del Primer Ministro Balladur. En Luxemburgo, donde las elecciones europeas coinciden con los comicios generales, la coalición entre socialistas y democristianos seguramente renovará su triunfo.

\section{La Campaña electoral en España}

Es en España donde el resultado de los comicios europeos se puede interpretar más en clave nacional que en el resto de los Estados europeos miembros. 
Para los oradores políticos predicar en esta campaña el mensaje europeo ha sido más difícil que en las convocatorias de 1987 y 1989, debido a una cierta crispación existente en el ambiente político y en la condición de test que los partidos mayoritarios han otorgado en las elecciones, en espera de ver cual es la reacción del pueblo español frente a la crisis económica y a los casos de corrupción que azotan al partido del Gobierno.

\section{A) Los Partidos y Coaliciones en Campaña}

Las pretensiones de las distintas formaciones políticas manifestadas en la campaña pueden ser resumidas de la siguiente forma:

Partido Socialista (PSOE). Se realizan estas consultas electorales en un mal momento para el partido del Gobierno, que se encuentra inmerso en un período de graves acusaciones de permisibilidad de corrupción. La crisis económica, que, aunque va cediendo no ofrece razones visibles para el electorado, ha producido peticiones de dimisión del Gobierno y solicitudes de planteamiento de cuestión de confianza con el fin de mostrar los apoyos con que el Gobierno cuenta en el Parlamento nacional.

En las elecciones al Parlamento europeo, el PSOE cuenta como mejor factor con su candidato, Fernando Morán, considerado por las encuestas electorales el candidato más apreciado por los electores ${ }^{1}$.

El Manifiesto europeo de 1994, programa electoral del PSOE, junto a ofrecer una recopilación de los logros en Europa del Gobierno, busca mostrar los objetivos del PSOE para la Conferencia Intergubernamental de 1996, dirigido, en primer lugar a la modificación de las Instituciones europeas, y desde el punto de vista económico persigue la modificación del sistema de financiación de la UE, en base a criterios más progresivos y solidarios que permitan una reducción en las cuotas españolas de participación.

Partido Popular (PP). Contrariamente al programa electoral del PSOE, el del Partido Popular muestra los reveses sufridos por el Gobierno socialista en europa. Además ha centrado su campaña en el ataque directo al Gobierno por la política económica desarrollada dentro de nuestras fronteras y por los escándalos de corrupción que nos han azotado, con el

1 El sondeo publicado por El País (5 de junio de 1994) ofrece una enorme ventaja sobre el Candidato popular. 
fin de aprovechar un momento propicio para aventajar un número de votos al PSOE, tal y como indican los sondeos electorales.

Su programa se centra, principalmente, en la necesidad de modificar la economía española con el fin de hacerla convergente a las nacesidades de la UE. La agricultura centra otro momento importante del programa del partido de la Oposición, atacando las cuotas lecheras y reivindicando determinadas actuaciones en el sector pesquero. Desde el punto de vista social, defiende un sistema más duro de protección a la familia y a la infancia.

Izquierda Unida (IU). Aunque el progrma de IU se aleja en varios aspectos del objeto de las elecciones, puede ser considerado como el más largo y detallado de los presentados ${ }^{2}$. Tras pedir la revisión del Tratado de Maastricht, solicita que éste se convierta en un auténtico proceso constituyente que corrija el déficit democrático de la UE, mediante el referéndum y el reconocimiento de una auténtica iniciativa legislativa del Parlamento europeo. Desde el punto de vista económico exige la modificación de los criterios de convergencia económica y el aumento del Presupuesto comunitario hasta el 16 del PIB.

Foro - CDS. Aunque los sondeos electorales pronostican la desaparición de esta coalición del Europarlamento, su campaña ha sido la más sosegada. Su programa va dirigido a una reforma de las Instituciones, buscando otorgar al Parlamento Europeo los mismos poderes del Consejo de Ministros. El segundo aspecto importante de la campaña centrista es el referente a la ecología y defensa del medio ambiente.

Convergencia i Unió (CIU). El partido que coopera con el PSOE en la gobernabilidad del pais ofrece un programa profundamente nacionalista. Defiende la participación de Gobiernos autónomos en las decisiones del Consejo de Ministros de la Unión, el fortalecimiento de las competencias del Comité de las Regiones, y la modificación del sistema electoral al Parlamento Europeo, de forma que se sustituya el sistema de circuscripción nacional por el de circuscripción regional.

Estas elecciones suponen una especie de test para CIU que pretende valorar de qué forma acepta el electorado catalán la pseudocoalición CIU-PSOE en el Gobierno nacional.

Partido Nacionalista Vasco (PNV). Con un marcado carácter europeista y un nacionalismo más vehemente que el de ClU, el PNV ofrece un 
programa dirigido a exigir la participación de representantes vascos en casi todas las Instituciones europeas con el fin de asegurar la defensa de los intereses vascos en Europa. Además solicita la posibilidad de que el Gobierno vasco pueda impugnar directamente ante el Tribunal de Justicia aquellas decisiones por las que pueda verse afectado.

\section{B) Sondeos electorales}

Todas las encuestas publicadas por los diarios de difusión nacional coinciden en tres aspectos: otorgan al PP la victoria en los comicios europeos; adjudican un importante incremento de votos y escaños a IU, y señalan un gran retroceso para el partido del Gobierno.

La máxima diferencia a favor del PP para las elecciones europeas la señala "El Periódico" ${ }^{3}$, con 10.2 puntos (40.7 frente a 30.6 del PSOE), siendo "Diario $16 "$ " quien ofrece la menor ventaja electoral para el partido de la oposición (32.1 frente a 30.3 del PSOE).

En 9.6 puntos sitúa «El Mundo" ${ }^{5}$ el triunfo del PP sobre el partido del Gobierno, frente a "La Vanguardia" ${ }^{6}$ que otorga una diferencia en torno a los 5 puntos. Entre estos dos sondeos $\mathrm{ABC}^{7}$ da una ventaja al PP de un 8 por $100, y$ "El País»" de un 63 por 100.

Por lo que respecta a IU, el porcentaje de votos oscila entre un 13.5 por 100 (El Pais) y el 15.2 por 100 que le otorga la encuesta publicada por "El Periódico", concediéndose un número de escaños entre 9 y 11.

Convergencia i Unió (CIU) mejora sus resultados pudiendo obtener entre 3 y 4 escaños frente a los 2 que consiguió en 1989. Convergencia $\mathrm{Na}$ cionalista, candidatura encabezada por Josu Jon Imaz (PNV) obtendría entre 2 y 3 escaños.

Otro dato de interés reflejado en los sondeos preelectorales supone la desaparición del Europarlamento de Foro - CDS, y de los restantes

3 Encuesta realizada por Vox Pública. El Periódico. 5 de junio de 1994.

Encuesta realizada por ICP-Research. Diario 16. 5 de junio de 1994.

Encuesta realizada por Sigma Dos. El Mundo. 5 de junio de 1994.

Encuesta realizada por Instituto Opina. La Vanguardia. 5 de junio de 1994.

Encuesta realizada por Gruppo. ABC. 5 de junio de 1994.

Encuesta realizada por Demoscopia. El País. 5 de junio de 1994. 
partidos y agrupaciones que en los comicios de 1989 obtuvieron 1 escaño, indicando una tendencia a la concentración del voto hacia los partidos mayoritarios.

\section{RESULTADOS ELECTORALES}

\section{Datos generales de Participación}

En lo que a participación se refiere, España alcanzó el segundo índice más alto de todos los países de la UE en las elecciones al Parlamento, por detrás tan solo de Alemania, ya que casi el 60 por 100 de los españoles acudieron a las urnas.

La abstención ha sido menor de la prevista, aunque la curva sigue una línea decreciente. Del baremo de participación quedan excluídos Bélgica, Grecia y Luxemburgo, ya que en estos Estados, el voto es obligatorio. También, el hecho de que en muchos Estados se haya hecho coincidir las Elecciones al Parlamento Europeo con comicios nacionales, generales o autonómicos, ha sido una de las causas que ha evitado una mayor tendencia al abstencionismo.

En los extremos de participación se encuentran Alemania, el Estado con mayor índice de participación, 60.8 por 100, aunque inferior a la de anteriores comicios europeos, y Gran Bretaña, donde el nivel de participación alcanzó tan solo el 36.2 por 100.

\section{Resultados por Estados}

Los conservadores británicos cosechan una fuerte derrota en estas elecciones europeas, con una pérdida de más de la mitad de los 32 escaños que tenían en Estrasburgo, a la par que los laboristas dan un gran paso, incluso en zonas que tradicionalmente se han considerado feudos conservadores.

En el país vecino, Portugal, los resultados ofrecen un escaso margen de diferencia entre los socialdemócratas y los socialistas, mientras que en Grecia, el PASOK se perfila como el vencedor de las elecciones en detrimento de los conservadores de Nueva Democracia. 
En Italia, Fuerza Italia, partido liderado por Silvio Berlusconi "vuelve a barrer" ${ }^{9}$. Los neofascistas de Alianza Nacional avanzan un punto respecto a las elecciones generales de marzo, y la Liga Norte, que no ha dejado de librar una guerra interna contra el Primer Ministro italiano, ha recibido un castigo electoral que debilita su posición de fuerza en el Gobierno.

Descensos socialistas se han producido en Bélgica y Francia, aunque el avance conservador es mayor en Francia. Los demoscristianos holandeses ganan y mejoran los resultados de 1989.

En Dimarca, salen del Parlamento los democristianos, aunque el resultado más importante lo obtienen los grupos antieuropeos que logran casi la cuarta parte de los escaños. En Irlanda, como era previsible no se producen novedades importantes.

Kohl Barre en Alemania, donde los liberales se quedan fuera de la Eurocámara y los Verdes logran el 10 por 100.

ALEMANIA

\begin{tabular}{cccccccc}
\hline & & \multicolumn{2}{c}{1989} & \multicolumn{2}{c}{1994} & \multicolumn{2}{c}{$1989-1994$} \\
\hline Partidos & Sondeos & Escaños & $\%$ & Escaños & $\%$ & Escaños & $\%$ \\
\hline SPD & 40 & 31 & 37,2 & 40 & 32,2 & +9 & $+5,1$ \\
\hline CDV & 48 & 25 & 29,5 & 39 & 32,0 & +24 & $+2,5$ \\
\hline FDP & - & 4 & 5,6 & - & 4,1 & $-1,5$ & \\
\hline CSU & - & 7 & 8,2 & 8 & 6,8 & +1 & $-1,4$ \\
\hline PDS & - & - & - & - & - & - & - \\
\hline V & 11 & 8 & 8,4 & 12 & 10,1 & +4 & $+1,7$ \\
\hline REP & - & 6 & 7,1 & - & 3,9 & -6 & $-3,2$ \\
\hline
\end{tabular}

Escaños: 99

Participación 1989: 60,3\%

1994: $60,1 \%$

$9 \quad$ El Mundo. 13 de junio de 1994. 
BÉLGICA

\begin{tabular}{cccccccc}
\hline & \multicolumn{3}{c}{1989} & \multicolumn{2}{c}{1994} & \multicolumn{2}{c}{$1989-1994$} \\
\hline Partidos & Sondeos & Escaños & $\%$ & Escaños & $\%$ & Escaños & $\%$ \\
\hline CVP & 4 & 5 & 21,1 & 4 & 17,1 & -1 & -4 \\
\hline SP & 3 & 3 & 12,4 & 3 & 11,0 & - & $-1,4$ \\
\hline VLD & 3 & 2 & 10,6 & 3 & 11,4 & +1 & $+0,8$ \\
\hline AGALEV & 1 & 1 & 7,6 & 1 & 6,7 & - & $-0,9$ \\
\hline PSC & 2 & 2 & 8,1 & 2 & 7,0 & - & $-1,1$ \\
\hline PS & 3 & 5 & 14,5 & 3 & 11,3 & -2 & $-3,2$ \\
\hline PRL-FOF & 3 & 2 & 7,2 & 3 & 9,0 & +1 & $+1,8$ \\
\hline ECOLO & 1 & 2 & 6,3 & 1 & 4,8 & -1 & $-1,5$ \\
\hline VU & 1 & 1 & 5,4 & 1 & 4,4 & - & -1 \\
\hline VB & 2 & 1 & 4,1 & 2 & 7,8 & +1 & $+3,9$ \\
\hline FN & 1 & - & - & 1 & 2,9 & +1 & $+2,9$ \\
\hline
\end{tabular}

Escaños: 25

Participación:

1989: $90,7 \%$

1994: $87,0 \%$

DINAMARCA

\begin{tabular}{cccccccc}
\hline & \multicolumn{3}{c}{1989} & \multicolumn{2}{c}{1994} & \multicolumn{2}{c}{$1989-1994$} \\
\hline Partidos & Sondeos & Escaños & $\%$ & Escaños & $\%$ & Escaños & $\%$ \\
\hline S & 3 & 4 & 23,3 & 3 & 15,8 & -1 & $-7,5$ \\
\hline FOLKEB & 1 & 4 & 18,9 & 2 & 10,3 & -2 & $-8,6$ \\
\hline V & 4 & 3 & 16,6 & 4 & 18,6 & +1 & +2 \\
\hline KF & 3 & 2 & 13,3 & 3 & 17,7 & +1 & $+4,3$ \\
\hline CD & - & 2 & 7,9 & - & 0,9 & -2 & -7 \\
\hline SF & 1 & 1 & 9,1 & 1 & 8,6 & - & $-0,5$ \\
\hline PR & 1 & - & - & 1 & 8,5 & +1 & $+8,5$ \\
\hline JUNIB & 3 & - & - & 2 & 15,2 & +2 & $+15,2$ \\
\hline
\end{tabular}

Escaños: 16

Participación: 
FRANCIA

\begin{tabular}{cccccccc}
\hline & \multicolumn{3}{c}{1989} & \multicolumn{2}{c}{1994} & \multicolumn{2}{c}{$1989-1994$} \\
\hline Partidos & Sondeos & Escaños & $\%$ & Escaños & $\%$ & Escaños & $\%$ \\
\hline UDF-RPR & 29 & 26 & 28,9 & 29 & 25,5 & +3 & $-3,4$ \\
\hline PS & 16 & 22 & 26,6 & 16 & 14,5 & -6 & $-12,1$ \\
\hline PCP & 7 & 7 & 7,7 & 6 & 6,9 & -1 & $-0,8$ \\
\hline FN & 10 & 10 & 11,7 & 10 & 10,5 & - & $-1,2$ \\
\hline V & - & 9 & 10,6 & - & - & - & - \\
\hline MRI & 12 & - & - & 13 & 12,1 & +13 & $+12,1$ \\
\hline LOE & 13 & - & - & 13 & 12,4 & +13 & $+12,4$ \\
\hline LS & - & - & - & - & - & - & - \\
\hline
\end{tabular}

Escaños: 87

Participación:

1989: $48,7 \%$

1994: $55,0 \%$

GRECIA

\begin{tabular}{cccccccc}
\hline & \multicolumn{3}{c}{1989} & \multicolumn{2}{c}{1994} & \multicolumn{2}{c}{$1989-1994$} \\
\hline Partidos & Sondeos & Escaños & $\%$ & Escaños & $\%$ & Escaños & $\%$ \\
\hline PASOK & 10 & 9 & 36,0 & 10 & 37,6 & +1 & $+1,6$ \\
\hline ND & 9 & 10 & 40,4 & 8 & 32,7 & -2 & $-7,4$ \\
\hline SAP & 2 & 4 & 14,3 & 2 & 6,2 & -2 & $-8,1$ \\
\hline DIANA & - & 1 & 1,4 & - & - & -1 & $-1,4$ \\
\hline PP & 2 & - & - & 3 & 8,7 & +3 & $+8,9$ \\
\hline KKE & 2 & - & - & 2 & 6,3 & +2 & $+6,3$ \\
\hline
\end{tabular}

Escaños: 25

Participación: $\quad 1989: 79,9 \%$

1994: $71,0 \%$ 
HOLANDA

\begin{tabular}{ccccccccc}
\hline & \multicolumn{3}{c}{1989} & \multicolumn{2}{c}{1994} & \multicolumn{2}{c}{$1989-1994$} \\
\hline Partidos & Sondeos & Escaños & $\%$ & Escaños & $\%$ & Escaños & $\%$ \\
\hline CDA & 10 & 10 & 34,6 & 10 & 30,8 & $=$ & $-3,8$ \\
\hline PVDA & 8 & 8 & 30,7 & 8 & 22,9 & $=$ & $-7,8$ \\
\hline VVD & 6 & 3 & 13,6 & 6 & 17,9 & +3 & $+4,6$ \\
\hline Regenboog & 1 & 2 & 7,0 & 1 & 3,7 & -1 & $-3,3$ \\
\hline SGP & 2 & 1 & 5,9 & 2 & 7,8 & +1 & $+1,9$ \\
\hline D'66 & 4 & 1 & 5,9 & 4 & 11,7 & +3 & $+5,8$ \\
\hline
\end{tabular}

Escaños: 31

Participación:

1989: $47,2 \%$

1994: $35,6 \%$

IRLANDA

\begin{tabular}{cccccccc}
\hline & & \multicolumn{2}{c}{1989} & \multicolumn{2}{c}{1994} & \multicolumn{2}{c}{$1989-1994$} \\
\hline Partidos & Sondeos & Escaños & $\%$ & Escaños & $\%$ & Escaños & $\%$ \\
\hline FF & 4 & 6 & 31,5 & 4 & 30,7 & -2 & $-0,8$ \\
\hline FG & 2 & 4 & 21,6 & 2 & 26,5 & -2 & $+4,9$ \\
\hline PD & - & 1 & 12,0 & - & - & - & - \\
\hline LAB & 1 & 1 & 9,5 & 1 & 11,5 & $=$ & $+2,0$ \\
\hline WP & - & 1 & 7,5 & - & - & - & - \\
\hline IND & - & 1 & 3,5 & - & - & - & - \\
\hline IND (V) & 1 & 1 & 3,3 & 1 & 9,6 & $=$ & $+6,3$ \\
\hline
\end{tabular}

Escaños: 15

Participación:

1989: $68,3 \%$

1994: $41,0 \%$ 
ITALIA

\begin{tabular}{cccccccc}
\hline & & \multicolumn{2}{c}{1989} & \multicolumn{2}{c}{1994} & \multicolumn{2}{c}{$1989-1994$} \\
\hline Partidos & Sondeos & Escaños & $\%$ & Escaños & $\%$ & Escaños & $\%$ \\
\hline FI & - & - & - & 27 & 30,6 & +27 & $+30,6$ \\
\hline LN & - & - & - & 6 & 6,6 & +6 & $+6,6$ \\
\hline AN & - & - & - & 11 & 12,5 & +11 & $+12,5$ \\
\hline PDS & - & 22 & 27,6 & 16 & 19,1 & -6 & $-8,5$ \\
\hline RC & - & - & - & 5 & 6,0 & +5 & $+6,0$ \\
\hline PSI/AD & - & 12 & 14,8 & 2 & 1,8 & -10 & -13 \\
\hline PPI & - & 26 & 32,9 & 8 & 10,0 & -18 & $-22,9$ \\
\hline LP & - & - & - & 2 & 2,1 & +2 & $+2,1$ \\
\hline CC & - & - & - & - & - & - & - \\
\hline ARC & - & 2 & 2,4 & 3 & 3,2 & +1 & $+0,8$ \\
\hline PRI & - & - & - & - & - & - & - \\
\hline PLI & - & 4 & 4,4 & - & - & -4 & $-4,4$ \\
\hline LR & - & - & - & - & - & - & - \\
\hline DP & - & 1 & 1,3 & 1 & 1,1 & - & $-0,2$ \\
\hline SVP & - & 1 & 0,5 & - & - & -1 & $-0,5$ \\
\hline UV/PSDA & - & 1 & 0,6 & - & - & -1 & $-0,6$ \\
\hline L.S. & - & - & - & 3 & 3,3 & +3 & $+3,3$ \\
\hline OTROS & - & - & - & 3 & 3,6 & +3 & $+3,6$ \\
\hline
\end{tabular}

Escaños: 87

Participación:

1989: $81,5 \%$

1994: $75,0 \%$ 


\section{LUXEMBURGO}

\begin{tabular}{cccccccc}
\hline & & \multicolumn{2}{c}{1989} & \multicolumn{2}{c}{1994} & \multicolumn{2}{c}{$1989-1994$} \\
\hline Partidos & Sondeos & Escaños & $\%$ & Escaños & $\%$ & Escaños & $\%$ \\
\hline PCS & 3 & 3 & 34,9 & 2 & 31,4 & -1 & $-3,5$ \\
\hline POSL & 2 & 2 & 25,4 & 2 & 24,8 & $=$ & $-0,6$ \\
\hline DP & 1 & 1 & 20,0 & 1 & 18,8 & $=$ & $-1,2$ \\
\hline Verde & - & - & - & 1 & 11,0 & +1 & $+11,0$ \\
\hline
\end{tabular}

Escaños: 6

Participación:

1989: $87,4 \%$

1994: $90,0 \%$

PORTUGAL

\begin{tabular}{cccccccc}
\hline & \multicolumn{4}{c}{1989} & \multicolumn{2}{c}{1994} & \multicolumn{2}{c}{$1989-1994$} \\
\hline Partidos & Sondeos & Escaños & $\%$ & Escaños & $\%$ & Escaños & $\%$ \\
\hline PSD & - & 9 & 32,7 & 9 & 34,3 & $=$ & $+1,6$ \\
\hline PS & - & 8 & 28,5 & 10 & 34,7 & +2 & $+6,2$ \\
\hline CDU & - & 4 & 14,4 & 3 & 11,2 & -1 & $-3,2$ \\
\hline CDS & - & 3 & 14,1 & 3 & 12,4 & $=$ & $-1,7$ \\
\hline
\end{tabular}

Escaños: 25

Participación:

1989: $51,2 \%$

1994: $37,5 \%$

REINO UNIDO

\begin{tabular}{cccccccc}
\hline & \multicolumn{3}{c}{1989} & \multicolumn{2}{c}{1994} & \multicolumn{2}{c}{$1989-1994$} \\
\hline Partidos & Sondeos & Escaños & $\%$ & Escaños & $\%$ & Escaños & $\%$ \\
\hline Laborista & 60 & 45 & 40,1 & 62 & 45,0 & +17 & $+4,0$ \\
\hline Conservador & 20 & 32 & 34,1 & 18 & 27,0 & -14 & $-7,1$ \\
\hline SNP & 2 & 1 & 2,7 & 2 & - & +1 & - \\
\hline DUP & - & 1 & 1,0 & 1 & - & $=$ & - \\
\hline SDLP & 1 & 1 & 0,8 & 1 & - & $=$ & - \\
\hline VUP & 1 & 1 & 0,7 & 1 & - & $=$ & - \\
\hline PLD & 2 & - & - & 2 & 16,0 & +2 & $+16,0$ \\
\hline
\end{tabular}

Escaños: 87

Participación: 


\section{Resultados e España}

\section{A) Resultados generales*}

\section{ESPAÑ̃}

\begin{tabular}{ccccccc}
\hline & \multicolumn{2}{c}{1989} & \multicolumn{2}{c}{1994} & \multicolumn{2}{c}{$1989-1994$} \\
\hline Partidos & Escaños & $\%$ & Escaños & $\%$ & Escaños & $\%$ \\
\hline PP & 15 & 21,41 & 28 & 40,20 & +13 & $+18,79$ \\
\hline PSOE & 27 & 39,57 & 22 & 30,67 & -5 & $-8,90$ \\
\hline IU & 4 & 6,06 & 9 & 13,46 & +5 & $+7,40$ \\
\hline CIU & 2 & 4,20 & 3 & 4,67 & +1 & $+0,47$ \\
\hline PEP & 1 & 1,51 & - & 1,29 & -1 & $-0,22$ \\
\hline CN & 1 & 1,91 & 2 & 2,81 & +1 & $+0,90$ \\
\hline Foro-CDS & 5 & 7,15 & - & 0,99 & -5 & $-7,14$ \\
\hline PA & 1 & 1,86 & - & 0,75 & -1 & $-1,11$ \\
\hline HB & 1 & 1,70 & - & 0,97 & -1 & $-0,63$ \\
\hline IP & 1 & 1,83 & - & - & - & - \\
\hline A. Ruiz M. & 2 & 3,84 & - & 0,44 & -2 & $-3,40$ \\
\hline
\end{tabular}

№ Electores:

№ Votantes:

31.558 .724

№ Votos en blanco:

18.664 .053

№ Votos nulos:

213.621

Escaños:

85.640 
B) Resultados por Partidos y Coaliciones

Partidos y Coaliciones de ámbito nacional

Partido Popular (PP). Los errores y las tragedias del Partido socialista han sido un material muy bien aprovechado por el PP ${ }^{10}$ que consigue su primera victoria en unos comicios convocados a nivel nacional con más de un $\mathbf{4 0}$ por 100 de los votos y 28 escaños, doblando casi los resultados obtenidos en 1989 (15 escaños). El triunfo del PP, basado en una alta fidelidad del voto en relación con los comicios generales del pasado año, supone, si se proyecta en los ámbitos local y autonómico un "terremoto en el mapa político" "puesto que ha logrado la victoria en 13 de las 17 CCAA $y$, en alguna de ellas ha obtenido la mayoría absoluta. Obtiene el voto urbano en una proporción muy superior al voto rural, ya que ha sido el partido más votado en 59 capitales de provincia y localidades con más de 100.000 habitantes.

El test al que se ha sometido, tanto el PP, como su líder, José María Aznar, en estas elecciones, se salda con un balance muy favorable.

Partido Socialista (PSOE). Diversas y fuertes reacciones provoca en el Aparato del partido el fuerte descenso del PSOE. A pesar de que, con el desgaste sufrido, cuenta con un gran apoyo (30 por 100 del electorado) ${ }^{12}$, el partido del Gobierno obtiene la mayor derrota sufrida hasta el momento.

La consulta electoral ha servido para materializar el voto de castigo contra el PSOE, que ha retrocedido en las elecciones europeas al mismo nivel porcentual de votos de 1979, perdiendo, incluso, en zonas que tradicionalmente le han dado el voto, aunque no ha aceptado el discurso derrotista del PP que ve en estos resultados asegurado el triunfo en las próximas citas con las urnas.

Izquierda Unida (IU). El pluralismo político en España queda asegurado con la firme presencia de IU. A la luz de los resultados se puede afirmar que la Coalición ha roto su techo electoral y apunta a la consolidación del proyecto mayoritario representado por Julio Anguita.

P. Calvo Hernández. "El PP se asoma al poder". El Mundo. 13 de junio

"J. Miguel Larraga. El País. 14 de Junio de 1994.

de 1994.

2 Julia Navarro. "El suelo de granito del PSOE». El Mundo. 13 de junio 
Con Alonso Puerta a la cabeza, IU ha batido un récord en la historia del comunismo postdemocrático, de forma que el viejo sueño de llegar a despistarle, incluso al PSOE, la hegemonía de la izquierda ${ }^{13}$, ya no parece una quimera inalcanzable.

El desgaste sufrido por el Gobierno produce una huída importante de votos socialistas hasta la coalición.

\section{Partidos y Coaliciones nacionalistas}

CIU ha mejorado sus resultados del 89, y obtiene un tercer escaño, sin que ello suponga un aumento de votos. El partido catalán triunfa en Cataluña, por primera vez, en unos comicios no autonómicos, donde el PSOE queda relegada como tercera fuerza política. Todo parece indicar que los catalanes han comprendido lo bueno que es tener un partido nacionalista con capacidad para negociar la estabilidad política del Gobierno. En el sentido malo de la balanza, indicar que en Cataluña, junto con Baleares, se registra el mayor índice de abstención.

Por su parte, los resultados en el País Vasco demuestran la consolidación de tendencias apuntadas en consultas anteriores y sugieren cambios significativos en el mapa electoral. La fortaleza del PNV queda demostrada de forma abierta, absorbiendo, cada vez más, gran número de votos de EA. Otro de los factores importantes es el gran retroceso, y la consiguiente desaparición del Parlamento Europeo, sufrida por $\mathrm{HB}$, que, no obstante, sigue siendo el partido más votado en Guipúzcoa. HB pierde 43.000 votos respecto a las elecciones generales, y se sitúa en su cuota más baja desde 1983.

En Navarra, las fuerzas nacionalistas evidencian una caída frente a UPN, versión navarra del PP, que obtiene el 40.8 por 100 de los votos.

\section{CONCLUSIONES}

En general, si excluímos los casos de Francia, España y Gran Bretaña, no ha habido un importante voto de castigo a los Gobiernos Nacionales. Sin embargo, el socialismo europeo ha sufrido un grave revolcón salvado, en parte, por el ascenso del laborismo inglés.

13 El Mundo. Editorial de 13 de junio de 1989. 
Aunque el Grupo Socialista será, probablemente, el primero en número de escaños, la derecha, aunque muy fragmentada, ha crecido mucho más que la izquierda. Tras estas elecciones, el mapa político de la UE aparece más fragmentado, de forma que el Parlamento refleja la variedad de situaciones políticas de cada uno de los "Doce", y recoge los frutos del duro proceso de ratificación del Tratado de Maastricht. Serán duras las negociaciones para la formación de los Grupos Parlamentarios.

Un dato importante a tener en cuenta es la aparición en el nuevo Parlamento de un buen número de grupos que militan abiertamente contra el Tratado de Maastricht, a lo que hay que añadir las formaciones italianas Forza Italia y Liga Norte, poco entusiastas con el proyecto europeo ${ }^{14}$. A pesar de estos últimos datos, las fuerzas europeistas siguen siendo mayoritarias en el Parlamento y este europeismo ha sufrido un importante respaldo con el fuerte apoyo conseguido en Austria por los partidos favorables a su entrada en la UE. 